\title{
On the existence of optimal meshes in every convex domain on the plane
}

\author{
András Kroó*† \\ Alfréd Rényi Institute of Mathematics \\ Hungarian Academy of Sciences \\ Budapest, Hungary \\ and \\ Budapest University of Technology and Economics \\ Department of Analysis \\ Budapest, Hungary
}

\begin{abstract}
In this paper we study the so called optimal polynomial meshes for domains in $K \subset \mathbb{R}^{d}, d \geq 2$. These meshes are discrete point sets $Y_{n}$ of cardinality $c n^{d}$ which have the property that $\|p\|_{K} \leq A\|p\|_{Y_{n}}$ for every polynomial $p$ of degree at most $n$ with a constant $A>1$ independent of $n$. It was conjectured earlier that optimal polynomial meshes exist in every convex domain. This statement was previously shown to hold for polytopes and $C^{2}$ like domains. In this paper we give a complete affirmative answer to the above conjecture when $d=2$.
\end{abstract}

\section{Introduction}

Consider the space $P_{n}^{d}$ of real algebraic polynomials in $d$ variables and total degree at most $n$. Given any compact subset $K \subset \mathbb{R}^{d}$ with nonempty interior we denote by $\|f\|_{K}:=\sup _{\mathbf{x} \in K}|f(\mathbf{x})|$ the usual supremum norm on $K$.

In this paper we will study the so called norming sets or admissible polynomial meshes introduced in [5] and [3]. The subsets $Y_{n} \subset K, n=1,2, \ldots$ are called norming sets in $K$ for $P_{n}^{d}, n=1,2, \ldots$ if there exists a constant $A>1$ independent of $n$ such that

$$
\|p\|_{K} \leq A\|p\|_{Y_{n}}, \quad \forall p \in P_{n}^{d}, n \in \mathbb{N} .
$$

*Supported by the OTKA Grant K111742. e-mail:kroo.andras@renyi.mta.hu, phone/fax: 3614838333

${ }^{\dagger}$ AMS Subject classification: 41A17. Key words and phrases: multivariate polynomials, tangential Bernstein inequalities, optimal meshes, convex bodies 
Of course the above definition is meaningful only if we try to keep the norming sets from being too large, i.e., one should aim for minimal possible cadinality $\# Y_{n}$. (Here and throughout this paper $\# B$ denotes the cardinality of the set $B$.) Clearly, we must have $\# Y_{n} \geq \operatorname{dim} P_{n}^{d}$ in order for (1) to hold.

Since $\operatorname{dim} P_{n}^{d} \sim n^{d}$ it follows that $n^{d}$ is the asymptotically optimal rate for the cardinality of norming sets for $P_{n}^{d}$. This leads to the following notion of optimal meshes introduced in [8]: polynomial norming sets $Y_{n} \subset K, n=1,2, \ldots$ satisfying (1) are called optimal meshes whenever $\# Y_{n} \leq B n^{d}, n \in \mathbb{N}$, with some $A, B>0$ depending only on $K$. Thus optimal meshes are norming sets for $P_{n}^{d}$ of cardinality $\sim n^{d}$.

Norming sets are applied, for instance for discrete least squares approximation, extracting discrete extremal sets of Fekete and Leja type, scattered data interpolation. Their study has received lately a considerable attention, see for instance [2], [4] where various applications and algorithms for their construction are discussed.

It should be also noted that norming sets give rise to good cubature formulas. Namely, as it was observed in [5] if $\phi \in C(K)^{*}$ is any linear functional of norm 1 on $C(K)$ and

$$
Y_{n}=\left\{y_{j n}: 1 \leq j \leq m_{n}\right\}, \quad \# Y_{n}=m_{n}, n \in \mathbb{N}
$$

are norming sets for $P_{n}^{d}$ satisfying (1) then there exist real numbers $c_{j n}, 1 \leq j \leq m_{n}$ so that the cubature formula

$$
\phi(p)=\sum_{j=1}^{m_{n}} c_{j n} p\left(y_{j n}\right), \quad \forall p \in P_{n}^{d}, n \in \mathbb{N}
$$

is exact on $P_{n}^{d}$, and $\sum_{j=1}^{m_{n}}\left|c_{j n}\right| \leq A, \forall n \in \mathbb{N}$. This ensures that the above cubature formula has good convergence properties in $C(K)$.

Finding exact geometric properties characterizing sets with optimal meshes turned out to be a rather difficult problem. It was conjectured in [8] that optimal meshes exist in every convex domain. This conjecture is known to hold for convex polytopes [8]. In [9] it was also shown that $C^{\alpha}$ star like domains with $\alpha>2-\frac{2}{d}$ possess optimal meshes. In particular, this implies existence of optimal meshes in $C^{2}$ star like domains. In the recent paper [10] the author verifies existence of optimal meshes for domains with so called "uniform double sided ball condition", which is an extension of the $C^{2}$ property. Note that all previous results on optimal meshes were given essentially for either the polytopes or some sort of $C^{2}$ like domains. Also it should be observed that these results were based on explicit construction of optimal meshes which typically takes into account the geometry of the boundary (with substantially more points needed in the neighborhood of non smooth parts of the boundary), and uniform distribution of points in the domain separated from the boundary.

On the other hand it was proved in [1] that any compact set $K \subset \mathbb{R}^{d}$ possesses a near optimal admissible polynomial mesh $Y_{n} \subset K, n=1,2, \ldots$ satisfying $\# Y_{n}=O\left((n \log n)^{d}\right), n \in \mathbb{N}$. However, contrary to previous results the proof of existence of near optimal meshes given in [1] is nonconstructive, it is based on Fekete points which in general can not be found explicitly.

In this paper we will settle completely the conjecture on existence of optimal meshes for arbitrary convex domains in $\mathbb{R}^{2}$.

Main Theorem. Every convex domain $K \subset \mathbb{R}^{2}$ possesses an optimal mesh. That is for any $0<\epsilon<1$ and $n \in \mathbb{N}$ there exist discrete sets $Y_{n} \subset K$ such that

$$
\|p\|_{K} \leq(1+\epsilon)\|p\|_{Y_{n}}, \quad \forall p \in P_{n}^{2}
$$


and

$$
\# Y_{n} \leq 4 \cdot 10^{5}\left(\frac{n}{\epsilon}\right)^{2}, n \in \mathbb{N}
$$

Let us point out the main new ideas need in the proof. It has been known for sometime, that tangential Bernstein type inequalities play an important role in the study of optimal meshes. Therefore we will consider the so called tangential Bernstein function (see (3) for the exact definition) which is known to be bounded on the boundary of $C^{2}$ star like domains and unbounded in general when $C^{2}$ smoothness does not hold (see [7]). The unboundness of the tangential Bernstein function is the major difficulty in constructing optimal meshes. In the present paper we will circumvent this difficulty by verifying the much deeper property of Lebesgue integrability of the tangential Bernstein function for regular convex domains. (Recall that a convex domain is called regular if it possesses a unique supporting hyperplane at every point of its boundary.) In order to show the Lebesgue integrability of the tangential Bernstein function we will apply Hardy-Littlewood maximal functions. Another important aspect of our approach consists in considering first regular convex domains and then passing to the general case by approximating arbitrary convex domains by regular ones. Of course, this approach can work only in case if our considerations are domain independent. We will achieve this domain independence by using the classical John ellipsoid theorem [6]. It also should be noted that our method below gives an explicit construction for optimal meshes based on the tangential Bernstein function.

\section{A tangential Bernstein type inequality}

Consider a regular convex domain $K \in \mathbf{R}^{2}$ which possesses a unique tangent line at every $\mathbf{z} \in \partial K$. Furthermore, let $D^{T} f(\mathbf{z}), \mathbf{z} \in \partial K$ denote the tangential derivative of $f$ in the unit tangential direction (with counter clockwise orientation).

The classical univariate Bernstein inequality states that for every algebraic polynomial $g$ of degree at most $n$

$$
\left|g^{\prime}(t)\right| \leq \frac{n}{\sqrt{(t-a)(b-t)}}\|g\|_{[a, b]}, \quad t \in(a, b) .
$$

Now we introduce the tangential Bernstein function of $K$ defined as

$$
B_{K}(\mathbf{z}):=\sup \left\{\frac{\left|D^{T} p(\mathbf{z})\right|}{\operatorname{deg} p}: p \in P^{2},\|p\|_{K} \leq 1\right\}, \quad \mathbf{z} \in \partial K,
$$

where $P^{2}:=\cup_{n} P_{n}^{2}$ stands for the space of all bivariate polynomials. In general, this is an unbounded function on $\partial K$. We will verify now the crucial fact that the tangential Bernstein function is Lebesgue integrable on the boundary $\partial K$. In addition, we will give a domain independent upper bound for its integral. In the sequel let $B(\mathbf{0}, r)$ denote balls in $\mathbb{R}^{2}$ of radius $r$ centered at the origin.

Theorem 1. Let $K \in \mathbb{R}^{2}$ be a regular convex domain such that $B(\boldsymbol{O}, 1) \subset K \subset B(\boldsymbol{O}, R)$ for some $R \geq 1$. Then

$$
\int_{\partial K} B_{K} d s \leq \eta R^{2}
$$


with an absolute constant $0<\eta<168(4 \pi+1)$.

First we shall need an auxiliary lemma on the Hardy-Littlewood maximal functions defined on finite intervals $I:=[a, b]$. Usually the Hardy-Littlewood maximal function is defined on all of $\mathbb{R}$, but we will need to adapt to the case of finite intervals. For $I=[a, b]$ denote by $I / 2:=$ $[(3 a+b) / 4,(3 b+a) / 4]$ and consider the Hardy-Littlewood maximal function of $f \in L^{1}(I), f \geq 0$ defined as

$$
M(f, x):=\sup _{0<h \leq(b-a) / 4} \frac{1}{2 h} \int_{x-h}^{x+h} f(u) d u, \quad x \in I / 2 .
$$

Lemma 1. For any $f \in L^{1}(I), f \geq 0$ on $I=[a, b]$ and $0<\alpha<1$ we have

$$
\int_{I / 2} M(f, x)^{\alpha} d x \leq \frac{5 \alpha}{1-\alpha} \int_{I} f(x) d x+\frac{b-a}{2} .
$$

Proof. Consider first the set

$$
E_{t}:=\{x \in I / 2: M(f, x)>t\}, t>0 .
$$

Then clearly for any $x \in E_{t}$ there exists $0<h_{x} \leq(b-a) / 4$ such that

$$
h_{x}<\frac{1}{2 t} \int_{x-h_{x}}^{x+h_{x}} f(u) d u .
$$

In particular, we also have that $E_{t} \subset \cup\left(x-h_{x}, x+h_{x}\right)$. Hence by the Vitali covering lemma the set $E_{t}$ possesses a countable cover $E_{t} \subset \cup_{j=1}^{\infty}\left(x_{j}-5 h_{x_{j}}, x_{j}+5 h_{x_{j}}\right), x_{j} \in E_{t}$ so that all intervals $\left(x_{j}-h_{x_{j}}, x_{j}+h_{x_{j}}\right) \subset I$ are disjoint. Therefore we have using (7)

$$
\mu\left(E_{t}\right) \leq 10 \sum_{j=1}^{\infty} h_{x_{j}} \leq \frac{5}{t} \sum_{j=1}^{\infty} \int_{x_{j}-h_{x_{j}}}^{x_{j}+h_{x_{j}}} f(x) d x \leq \frac{5}{t} \int_{I} f(x) d x
$$

where $\mu$ stands for the Lebesgue measure on the real line. Furthermore estimate (8) yields

$$
\begin{gathered}
\int_{1}^{\infty} \mu\left\{x \in I / 2: M(f, x)>t^{1 / \alpha}\right\} d t \leq \int_{1}^{\infty} \frac{5}{t^{1 / \alpha}} \int_{I} f(x) d x d t \\
=5 \int_{I} f(x) d x \int_{1}^{\infty} \frac{1}{t^{1 / \alpha}} d t=\frac{5 \alpha}{1-\alpha} \int_{I} f(x) d x .
\end{gathered}
$$

On the other hand applying the Cavalieri principle to the same integral we obtain

$$
\begin{gathered}
\int_{1}^{\infty} \mu\left\{x \in I / 2: M(f, x)^{\alpha}>t\right\} d t=\int_{1}^{\infty} \int_{I / 2} \chi_{\left\{x \in I / 2: M(f, x)^{\alpha}>t\right\}} d x d t= \\
\int_{I / 2} \int_{1}^{\infty} \chi_{\left\{x \in I / 2: M(f, x)^{\alpha}>t\right\}} d t d x=\int_{\{x \in I / 2: M(f, x)>1\}}\left(M(f, x)^{\alpha}-1\right) d x=\int_{E_{1}} M(f, x)^{\alpha} d x-\mu\left(E_{1}\right) .
\end{gathered}
$$

Evidently, the last two relations combined yield

$$
\int_{I / 2} M(f, x)^{\alpha} d x \leq \int_{E_{1}} M(f, x)^{\alpha} d x+\mu\left(I / 2 \backslash E_{1}\right)
$$




$$
\leq \frac{5 \alpha}{1-\alpha} \int_{I} f(x) d x+\mu\left(I / 2 \backslash E_{1}\right)+\mu\left(E_{1}\right)=\frac{5 \alpha}{1-\alpha} \int_{I} f(x) d x+\frac{b-a}{2} .
$$

Proof of Theorem 1. The convex domain $K$ can be represented in the form

$$
K:=\left\{(x, y) \in \mathbb{R}^{2}: a \leq x \leq b, f(x) \leq y \leq g(x)\right\},
$$

where $[-1,1] \subset[a, b] \subset[-R, R]$. We will assume first that $f(x), g(x)$ have absolutely continuous derivatives on $[a, b]$ and call the convex domain $K$ an $\mathrm{AC}$ convex domain in this case. Since $B(\mathbf{0}, 1) \subset K$ it easily follows by the convexity of $K$ that any tangent to $K$ can intersect axis $x$ only outside $(-1,1)$, i.e., $(1-|x|)\left|f^{\prime}(x)\right| \leq|f(x)|, x \in(-1,1)$. In addition, $K \subset B(\mathbf{0}, R)$ which yields

$$
\left|f^{\prime}(x)\right| \leq \frac{|f(x)|}{1-|x|} \leq \frac{R}{1-|x|}, \quad \forall|x|<1
$$

Choose any $\left|x_{0}\right| \leq 1 / 4$. Since $B(\mathbf{0}, 1) \subset K$ we have that $f(x) \leq-\sqrt{1-x^{2}} \leq 0, x \in(-1,1)$. Using that $f\left(x_{0}\right) \leq 0$ and $f^{\prime \prime} \geq 0$ a.e. we have by the Taylor formula for any $\left|x_{0}-x\right| \leq 1 / 4$

$$
f(x)=f\left(x_{0}\right)+f^{\prime}\left(x_{0}\right)\left(x-x_{0}\right)+\int_{x_{0}}^{x} f^{\prime \prime}(t)(x-t) d t \leq f^{\prime}\left(x_{0}\right)\left(x-x_{0}\right)+2\left(x-x_{0}\right)^{2} M\left(f^{\prime \prime}, x_{0}\right),
$$

where

$$
M\left(f^{\prime \prime}, x\right):=\sup _{0<h \leq 1 / 4} \frac{1}{2 h} \int_{x-h}^{x+h} f^{\prime \prime}(t) d t, \quad x \in I / 2
$$

stands for the Hardy-Littlewood maximal function (5) of $f^{\prime \prime}$ defined for $I:=[-1 / 2,1 / 2]$. Hence setting

$$
p_{2}(x):=f^{\prime}\left(x_{0}\right)\left(x-x_{0}\right)+2\left(x-x_{0}\right)^{2} M\left(f^{\prime \prime}, x_{0}\right) \in P_{2}^{1}
$$

it follows by (10) that

$$
f(x) \leq p_{2}(x) \leq \frac{4 R}{3}\left|x_{0}-x\right|+2\left(x-x_{0}\right)^{2} M\left(f^{\prime \prime}, x_{0}\right), \quad\left|x_{0}-x\right| \leq 1 / 4 .
$$

On the other hand using again that $B(\mathbf{0}, 1) \subset K$ we also have that $g(x) \geq \frac{\sqrt{3}}{2}$ whenever $|x| \leq 1 / 2$. In view of the previous estimate this means that setting

$$
A:=\frac{1}{4\left(R+M\left(f^{\prime \prime}, x_{0}\right)^{1 / 2}\right)} \leq \frac{1}{4}
$$

yields

$$
f(x) \leq p_{2}(x) \leq g(x), \quad\left|x_{0}-x\right| \leq A .
$$

Hence recalling representation (9) we obtain

$$
\left(x, p_{2}(x)\right) \subset K \text {, whenever }\left|x_{0}-x\right| \leq A .
$$

Now let $p \in P_{n}^{2}$ be a bivariate polynomial of degree $n$ such that $\|p\|_{K}=1$. Then by the last observation it follows that the univariate polynomial $q(x):=p\left(x, p_{2}(x)\right) \in P_{2 n}^{1}$ satisfies

$$
|q(x)| \leq 1, \quad\left|x_{0}-x\right| \leq A .
$$


Hence using the classical Bernstein inequality (2) for the univariate polynomial $q$ of degree $\leq 2 n$ with $[a, b]:=\left[x_{0}-A, x_{0}+A\right]$ yields

$$
\left|q^{\prime}\left(x_{0}\right)\right| \leq \frac{2 n}{A}=8 n\left(R+M\left(f^{\prime \prime}, x_{0}\right)^{1 / 2}\right) .
$$

On the other hand for $\mathbf{z}_{0}:=\left(x_{0}, f\left(x_{0}\right)\right) \in \partial K$ we have using that $p_{2}^{\prime}\left(x_{0}\right)=f^{\prime}\left(x_{0}\right)$

$$
\left(1+f^{\prime}\left(x_{0}\right)^{2}\right)^{1 / 2} D^{T} p\left(\mathbf{z}_{0}\right)=\left(p_{x}\left(\mathbf{z}_{0}\right)+f^{\prime}\left(x_{0}\right) p_{y}\left(\mathbf{z}_{0}\right)\right)=q^{\prime}\left(x_{0}\right) .
$$

The last two relations yield that

$$
\left(1+f^{\prime}\left(x_{0}\right)^{2}\right)^{1 / 2}\left|D^{T} p\left(\mathbf{z}_{0}\right)\right|=\left|q^{\prime}\left(x_{0}\right)\right| \leq 8 n\left(R+M\left(f^{\prime \prime}, x_{0}\right)^{1 / 2}\right),
$$

i.e.,

$$
\left(1+f^{\prime}(x)^{2}\right)^{1 / 2} B_{K}(x, f(x)) \leq 8\left(R+M\left(f^{\prime \prime}, x\right)^{1 / 2}\right), \quad \forall|x| \leq \frac{1}{4} .
$$

Now we can apply Lemma 1 for the function $f^{\prime \prime} \in L^{1}(I)$ with $I:=[-1 / 2,1 / 2]$ and $\alpha=\frac{1}{2}$. Hence by (11) and (6) we have

$$
\int_{I / 2}\left(1+f^{\prime}(x)^{2}\right)^{1 / 2} B_{K}(x, f(x)) d x \leq \int_{I / 2} 8\left(R+M\left(f^{\prime \prime}, x\right)^{1 / 2}\right) d x \leq 4 R+4+40 \int_{I} f^{\prime \prime}(x) d x .
$$

Thus denoting by $\gamma$ the arc on $\partial K$ corresponding to the interval $I / 2$ we obtain by (12) and (10)

$$
\int_{\gamma} B_{K} d s \leq 4 R+4+40\left(f^{\prime}(1 / 2)-f^{\prime}(-1 / 2) \leq 164 R+4 \leq 168 R .\right.
$$

Now we need to realize how many of those arcs are needed to cover $\partial K$. Since length of any circular arc of radius $R$ corresponding to $I / 2$ is at least $\frac{1}{2}$, it is clear that $\partial B(\mathbf{0}, R)$ can be covered by at most $[4 \pi R]+1$ of such arcs. Recalling that $K \subset B(\mathbf{0}, R)$ it follows that $\partial K$ also can be covered by at most $[4 \pi R]+1$ arcs corresponding to intervals of length $\frac{1}{2}$, i.e. using (13) and rotation invariance we finally arrive at the upper bound

$$
\int_{\partial K} B_{K} d s \leq([4 \pi R]+1) 168 R \leq \eta R^{2}
$$

with an absolute constant $0<\eta<168(4 \pi+1)$.

Thus we obtained the required estimate for any AC convex domain $K \subset \mathbb{R}^{2}$. We will extend now the statement to any regular convex domain $K$ by approximating it in the Hausdorff metric by AC convex domains $K_{m} \rightarrow K$. It is crucial to note that the above upper bound for AC convex domains is domain independent, this makes the approximation procedure possible. Namely, given a regular convex domain $K=\left\{r(t) e^{i t}, 0 \leq t \leq 2 \pi\right\}$ with some continuously differentiable radial function $r(t)$ we can approximate it by convex domains $K_{m}=\left\{r_{m}(t) e^{i t}, 0 \leq t \leq 2 \pi\right\}, K \subset K_{m}$ with absolutely continuous radial derivatives $r_{m}^{\prime}$ so that $r_{m}, r_{m}^{\prime}$ uniformly converge to $r, r^{\prime}$, respectively. One way to accomplish this is the following procedure. Set $A_{j}:=r\left(t_{j}\right) e^{i t_{j}}$ with $t_{j}:=2 \pi j / m, 1 \leq j \leq m$, and consider the tangent lines to $\partial K$ at each of $A_{j}, 1 \leq j \leq m$. This lines define a convex polygon containing $K$ with some vertices $B_{j}$. Furthermore, into each angle with vertex $B_{j}$ inscribe small 
circles in order to smoothen the polygon. This will result in convex domains $K_{m}$ with properties stated above. Denote by $B_{n, K}$ the $n$-th order tangential Bernstein function when the sup in (3) is taken over polynomials of degree at most $n$. Then clearly, we have by continuity and compactness of the unit ball in finite dimensional spaces that uniformly on $\partial K$

$$
B_{n, K_{m}} \rightarrow B_{n, K}, \quad m \rightarrow \infty, \forall n .
$$

Now using the bound (4) for each $K_{m}$ this obviously implies that

$$
\int_{\partial K} B_{n, K} d s \leq \eta R^{2}, \quad \forall n
$$

Since the functions $B_{n, K}$ monotonously increase to $B_{K}$ the statement of the theorem follows from the last estimate by the Levi monotone convergence theorem.

\section{Proof of the Main Theorem}

The construction of optimal meshes consists of two basic steps. At first given a convex body $K \subset \mathbb{R}^{2}$ and $\mathbf{c} \in \operatorname{Int} K$ we consider the level curves

$$
K_{j}:=\left\{t_{j}(\mathbf{z}-\mathbf{c})+\mathbf{c}: \mathbf{z} \in \partial K\right\}, 0 \leq j \leq N
$$

which are dilations by $t_{j}$ of the boundary $\partial K$ with respect to the center $\mathbf{c}$. Then we choose properly the center $\mathbf{c}$ and $0=t_{1}<t_{2}<\ldots<t_{N}=1$ with $N \sim n$ so that with some $0<a<1$ chosen arbitrarily small

$$
(1-a)\|p\|_{K} \leq \max _{0 \leq j \leq N}\|p\|_{K_{j}}, \quad \forall p \in P_{n}^{2} .
$$

After this is accomplished using Theorem 1 we will solve the more delicate problem of finding discrete point sets $Y_{n} \subset \partial K, \# Y_{n} \sim n$ such that for $\forall p \in P_{n}^{2}$ with $\|p\|_{K}=\|p\|_{\partial K}$ we have

$$
\|p\|_{K} \leq(1+a)\|p\|_{Y_{n}} .
$$

Then by the affine invariance it will follow that the last estimate holds for dilations of these discrete sets on every level curve $K_{j}, 0 \leq j \leq N$. Finally, combining the two steps above yields the desired optimal mesh.

The next lemma provides a choice of proper $0=t_{1}<t_{2}<\ldots<t_{N}=1$ needed in the first step of the construction.

Lemma 2. Let $K \in \mathbb{R}^{2}$ be a convex domain. Then there exist $\boldsymbol{c} \in \operatorname{IntK}$ and $0=t_{1}<t_{2}<\ldots<$ $t_{m n}=1, n \in \mathbb{N}, m \geq 3$ such that we have for the level curves $K_{j}$ given by (14)

$$
\left(1-\frac{2}{m}\right)\|p\|_{K} \leq \max _{0 \leq j \leq m n}\|p\|_{K_{j}}, \quad \forall p \in P_{n}^{d}, \quad n \in \mathbb{N}, m \geq 3 .
$$

Proof. By the classical John maximal ellipsoid theorem [6] for any convex body $K \subset \mathbb{R}^{d}$ there exists a unique ellipsoid $E$ of maximal volume and center $\mathbf{c}$ such that $E \subset K \subset \mathbf{c}+d(E-\mathbf{c})$ where 
$T(E)=B(\mathbf{0}, 1)$ with some regular affine map $T: \mathbb{R}^{d} \rightarrow \mathbb{R}^{d}$. We may assume $\mathbf{c}=\mathbf{0}$. Then setting $G:=T(K)$ we have $B(\mathbf{0}, 1) \subset G \subset B(\mathbf{0}, d)$. Since the statement of the lemma is affine invariant we may assume without the loss of generality that $K \subset \mathbb{R}^{2}$ is such that $B(\mathbf{0}, 1) \subset K \subset B(\mathbf{0}, 2)$.

Let $\|p\|_{K}=|p(\mathbf{x})|, \mathbf{x} \in K$. Furthermore, assume that $\mathbf{a}, \mathbf{b} \in \partial K, 1 \leq|\mathbf{a}| \leq|\mathbf{b}| \leq 2$ are the points where the line passing through $\mathbf{0}, \mathbf{x}$ intersects the boundary of $K$. Then clearly $\mathbf{x}=$ $u \mathbf{a}+(1-u) \mathbf{b}$ and $\mathbf{b}=-s \mathbf{a}$ for some $u \in[0,1], s \in[1,2]$.

Set

$$
g(t):=p(\mathbf{a} t) \in P_{n}^{1}
$$

Then it is easy to see that,

$$
\|p\|_{K}=|p(\mathbf{x})|=\|g\|_{[-s, 1]} .
$$

Hence using the univariate Bernstein inequality (2) on the interval $[-s, 1]$ yields

$$
\left|g^{\prime}(t)\right| \leq \frac{n}{\sqrt{(t+s)(1-t)}}\|g\|_{[-s, 1]}, \quad t \in(-s, 1) .
$$

Now we shall distinguish between two cases.

Case 1. $\mathbf{x} \in[\mathbf{0}, \mathbf{a}]$, i.e., $\|g\|_{[-s, 1]}=\|g\|_{[0,1]}$. Then set

$$
g(t)=g(\cos \phi):=q(\phi), \quad t=\cos \phi, \phi \in[0, \pi / 2] .
$$

Evidently, it follows from (16) that whenever $\phi \in[0, \pi / 2]$

$$
\left|q^{\prime}(\phi)\right|=\left|g^{\prime}(t) \sin \phi\right| \leq \frac{n\|g\|_{[-s, 1]} \sin \phi}{\sqrt{1-\cos \phi}} \leq \sqrt{2} n\|g\|_{[0,1]}=\sqrt{2} n\|q\|_{[0, \pi / 2]} .
$$

Furthermore, setting

$$
\phi_{j}:=\frac{j \pi}{2 m n}, \quad t_{j}:=\cos \phi_{j}, \quad 0 \leq j \leq m n
$$

it follows that

$$
q\left(\phi_{j}\right)=g\left(t_{j}\right), \quad \mathbf{a} t_{j} \in K_{j}=\left\{t_{j} \mathbf{z}: \mathbf{z} \in \partial K\right\}, \quad 0 \leq j \leq m n
$$

and thus

$$
\max _{0 \leq j \leq m n}\|p\|_{K_{j}} \geq \max _{0 \leq j \leq m n}\left|g\left(t_{j}\right)\right|=\max _{0 \leq j \leq m n}\left|q\left(\phi_{j}\right)\right| .
$$

Since $\phi_{j}:=\frac{j \pi}{2 m n}$ are equidistributed in $[0, \pi / 2]$ with step $\frac{\pi}{2 m n}$ for any $\phi^{*} \in[0, \pi / 2]$ such that

$$
\|g\|_{[-s, 1]}=\|g\|_{[0,1]}=\|q\|_{[0, \pi / 2]}=\left|q\left(\phi^{*}\right)\right|
$$

we can find a $\phi_{k}$ so that $\left|\phi_{k}-\phi^{*}\right| \leq \frac{\pi}{4 m n}$. Hence and by (17)

$$
\|q\|_{[0, \pi / 2]}-\max _{0 \leq j \leq m n}\left|q\left(\phi_{j}\right)\right| \leq\left|q\left(\phi^{*}\right)-q\left(\phi_{k}\right)\right| \leq \frac{\sqrt{2} \pi}{4 m}\|q\|_{[0, \pi / 2]} \leq \frac{2}{m}\|q\|_{[0, \pi / 2]} .
$$

Evidently combining this with (18) yields that

$$
\max _{0 \leq j \leq m n}\|p\|_{K_{j}} \geq \max _{0 \leq j \leq m n}\left|q\left(\phi_{j}\right)\right| \geq\left(1-\frac{2}{m}\right)\|q\|_{[0, \pi / 2]} .
$$


Since

$$
\|q\|_{[0, \pi / 2]}=\|g\|_{[0,1]}=\|g\|_{[-s, 1]}=\|p\|_{K}
$$

the last estimate implies (15).

Case 2. $\mathbf{x} \in[\mathbf{0}, \mathbf{b}]$, i.e., $\|g\|_{[-s, 1]}=\|g\|_{[-s, 0]}$. Now we set

$$
g(t)=g(s \cos (\phi)):=q(\phi), \quad t=s \cos \phi \in[-s, 0], \phi \in[\pi / 2, \pi] .
$$

Using again the Bernstein inequality (16) yields with $t=s \cos \phi \in[-s, 0]$

$$
\left|q^{\prime}(\phi)\right|=\left|g^{\prime}(t) s \sin \phi\right| \leq \frac{n \sqrt{s^{2}-t^{2}}}{\sqrt{(t+s)(1-t)}}\|g\|_{[-s, 1]} \leq \sqrt{s} n\left\|_{g}\right\|_{[-s, 0]} \leq \sqrt{2} n\left\|_{q}\right\|_{[\pi / 2, \pi]}, \quad \phi \in[\pi / 2, \pi] .
$$

Now we consider $\phi_{j}:=\pi-\frac{j \pi}{2 m n}, 0 \leq j \leq m n$. It follows that

$$
q\left(\phi_{j}\right)=g\left(s \cos \left(\pi-\frac{j \pi}{2 m n}\right)\right)=g\left(-s t_{j}\right), \mathbf{b} t_{j}=-s t_{j} \mathbf{a} \in K_{j}, 0 \leq j \leq m n
$$

and thus

$$
\max _{0 \leq j \leq m n}\|p\|_{K_{j}} \geq \max _{0 \leq j \leq m n}\left|g\left(-s t_{j}\right)\right|=\max _{0 \leq j \leq m n}\left|q\left(\phi_{j}\right)\right| .
$$

Moreover, in this case $\phi_{j}:=\pi-\frac{j \pi}{2 m n}$ are equidistributed in $[\pi / 2, \pi]$ with step $\frac{\pi}{2 m n}$. In addition, (20) and (19) provide the same estimates as (18) and (17), respectively. Now the proof can be completed analogously to Case 1.

Lemma 3. Let $0<a<\frac{1}{2}$ and consider a regular convex domain $K \in \mathbb{R}^{2}$ with $B(\boldsymbol{O}, 1) \subset K \subset$ $B(\boldsymbol{O}, R)$. Then there exist discrete point sets

$$
Y_{n} \subset \partial K, \text { with } \# Y_{n} \leq \frac{\eta R^{2}+1}{a} n, n \in \mathbb{N},
$$

with $0<\eta<168(4 \pi+1)$ so that whenever $p \in P_{n}^{2}$ and $(1-a)\|p\|_{K} \leq\|p\|_{\partial K}$ we have

$$
(1-a)^{2}\|p\|_{K} \leq\|p\|_{Y_{n}}
$$

Proof. First let us note that we can assume that $\|p\|_{K}=1,\|p\|_{\partial K} \geq 1-a$. Consider any two points $\mathbf{z}_{1}, \mathbf{z}_{2} \in \partial K$. The boundary of $K$ possesses a natural parametrization $\partial K=(x(s), y(s))$ with some differentiable functions $x(s), y(s), 0 \leq s \leq L$, where $L$ stands for the length of the boundary.

Then setting $Q(s):=p(x(s), y(s))$ it easily follows that

$$
\left|p\left(\mathbf{z}_{1}\right)-p\left(\mathbf{z}_{2}\right)\right|=\left|Q\left(s_{1}\right)-Q\left(s_{2}\right)\right|=\left|\int_{s_{1}}^{s_{2}} Q^{\prime}(s) d s\right| \leq \int_{s_{1}}^{s_{2}}\left|D^{T} p(\mathbf{z})\right| d s \leq n \int_{s_{1}}^{s_{2}} B_{K}(x(s), y(s)) d s .
$$

Consider now the strictly monotone increasing function

$$
\Theta(s):=\int_{0}^{s} B_{K}(x(\tau), y(\tau)) d \tau, \quad 0 \leq s \leq L .
$$

Then by the last estimate

$$
\left|p\left(\mathbf{z}_{1}\right)-p\left(\mathbf{z}_{2}\right)\right| \leq n\left|\Theta\left(s_{2}\right)-\Theta\left(s_{1}\right)\right|
$$


Now with an arbitrary $m \in \mathbb{N}$ to be specified below choose $0 \leq s_{j} \leq L$ so that

$$
\Theta\left(s_{j}\right)=\frac{\Theta(L) j}{m n}, \quad 0 \leq j \leq m n .
$$

Let $\mathbf{z}_{0}=\left(x\left(s_{0}\right), y\left(s_{0}\right)\right) \in \partial K, 0 \leq s_{0} \leq L$ be such that $\left|p\left(\mathbf{z}_{0}\right)\right|=\|p\|_{\partial K} \geq 1-a$. Since $\Theta\left(s_{j}\right)$ are equidistributed in $[0, \Theta(L)]$ with step $\frac{\Theta(L)}{m n}$ it follows that for some $0 \leq k \leq m n$ we have

$$
\left|\Theta\left(s_{k}\right)-\Theta\left(s_{0}\right)\right| \leq \frac{\Theta(L)}{2 m n} .
$$

Now set

$$
Y_{n}:=\left\{\mathbf{y}_{j}:=\left(x\left(s_{j}\right), y\left(s_{j}\right)\right): 0 \leq j \leq m n\right\} \subset \partial K .
$$

Note that by Theorem $1 \Theta(L) \leq \eta R^{2}$. Then by (22) and (23)

$$
1-a-\left|p\left(\mathbf{y}_{k}\right)\right| \leq\left|p\left(\mathbf{z}_{0}\right)-p\left(\mathbf{y}_{k}\right)\right| \leq n\left|\left(\Theta\left(s_{0}\right)-\Theta\left(s_{k}\right)\right)\right| \leq \frac{\Theta(L)}{2 m} \leq \frac{\eta R^{2}}{2 m} .
$$

Thus we obtain setting $m:=\left[\frac{\eta R^{2}}{a}\right]+1$

$$
\|p\|_{Y_{n}} \geq\left|p\left(\mathbf{y}_{k}\right)\right| \geq 1-a-\frac{\eta R^{2}}{2 m} \geq(1-a)^{2}\|p\|_{K}
$$

Evidently, $\# Y_{n}=m n+1 \leq \frac{\eta R^{2}+1}{a} n$ which completes the proof of the lemma.

Proof of the Main Theorem. As it was mentioned above the statement of the main theorem is affine invariant. So by the John maximal ellipsoidal theorem [6] we may again restrict our attention to convex domains $K \subset \mathbb{R}^{2}$ such that $B(\mathbf{0}, 1) \subset K \subset B(\mathbf{0}, 2)$. We will prove the main theorem first for regular convex domains $K$ with the above property, and then use a compactness type argument in order to extend it to arbitrary convex domains.

Consider any $0<\epsilon<1$. We will apply now Lemma 2 with $m:=[8 / \epsilon]+1$. Thus there exist level curves $K_{j}, 0 \leq j \leq m n$ for which we have

$$
\left(1-\frac{\epsilon}{4}\right)\|p\|_{K} \leq\left(1-\frac{2}{m}\right)\|p\|_{K} \leq \max _{0 \leq j \leq m n}\|p\|_{K_{j}}, \quad \forall p \in P_{n}^{2}
$$

On each level curve $K_{j}$ we shall choose discrete points using Lemma 3. Note that the level curves $K_{j}$ are dilations of $\partial K$ and since Lemma 3 is dilation invariant we can apply it on each of the level curves $K_{j}$ with $R:=2$ and $a:=\epsilon / 4$. This yields discrete point sets $Y_{j, n} \subset K_{j}$ such that whenever $p \in P_{n}^{2}$ and

$$
\left(1-\frac{\epsilon}{4}\right)\|p\|_{K} \leq\|p\|_{K_{j}}
$$

we have

$$
\left(1-\frac{\epsilon}{4}\right)^{2}\|p\|_{K_{j}} \leq\|p\|_{Y_{j, n}}, \quad 0 \leq j \leq m n
$$

where $\# Y_{j, n} \leq \frac{c n}{\epsilon}, \quad 0 \leq j \leq m n$ with $c<4 \cdot 10^{4}$. 
Now we set $Y_{n}:=\cup_{0 \leq j \leq m n} Y_{j, n}$. Then using that $m:=[8 / \epsilon]+1$ we clearly have by the last estimate

$$
\# Y_{n} \leq(m n+1) \frac{c n}{\epsilon}<4 \cdot 10^{5}\left(\frac{n}{\epsilon}\right)^{2} .
$$

Furthermore, it follows from (24) that whenever $p \in P_{n}^{2}$ we have for some $0 \leq i \leq m n$

$$
\left(1-\frac{\epsilon}{4}\right)\|p\|_{K} \leq\|p\|_{K_{i}}
$$

Therefore estimate (25) must hold with $j:=i$. Thus we obtain by (27) and (25)

$$
(1-\epsilon)\|p\|_{K} \leq\left(1-\frac{\epsilon}{4}\right)^{3}\|p\|_{K} \leq\left(1-\frac{\epsilon}{4}\right)^{2}\|p\|_{K_{i}} \leq\|p\|_{Y_{i, n}} \leq\|p\|_{Y_{n}}, \quad \forall p \in P_{n}^{2}
$$

Thus we arrived at the required estimate with the discrete point sets $Y_{n}$ having the needed cardinality. This completes the proof of the theorem for the case of regular convex bodies.

Now based on the fact that (26) provides a domain independent bound for the cardinality of the optimal mesh for any regular convex domain we can finish the proof of the main theorem in general case using a standard compactness type argument. Thus let $K \subset \mathbb{R}^{2}$ be an arbitrary convex domain and fix an integer $n \in \mathbb{N}$ and $0<\epsilon<1$. We can approximate $K$ by a sequence of imbedded regular convex domains $K_{m} \subset K, K_{m} \rightarrow K, m \rightarrow \infty$ in the Hausdorff metric. In view of the existence of optimal meshes with required cardinality for regular convex domains $K_{m}$ there exist discrete sets $Y_{m}:=\left\{y_{j, m}, 1 \leq j \leq N_{m}\right\} \subset K_{m} \subset K$ satisfying (26) such that

$$
\|p\|_{K_{m}} \leq(1+\epsilon)\|p\|_{Y_{m}}, \quad \forall p \in P_{n}^{2} .
$$

Moreover, because of the domain independent nature of the upper bound (26) for cardinality of each $Y_{m}$ we may assume without loss of generality, that

$$
\# Y_{m}=N_{m}=N:=\left[4 \cdot 10^{5}\left(\frac{n}{\epsilon}\right)^{2}\right], \forall m \in \mathbb{N} .
$$

Now for every $1 \leq j \leq N$ the sequence of points $\left\{y_{j, m}, m=1,2, \ldots\right\}$ has a convergent subsequence in $K$, so passing if necessary to subsequences step by step we can assume that $Y_{m} \rightarrow Y, m \rightarrow \infty$ where $Y \subset K$ also satisfies $\# Y \leq N$. Then evidently we have that

$$
\|p\|_{Y_{m}} \rightarrow\|p\|_{Y}, \quad\|p\|_{K_{m}} \rightarrow\|p\|_{K}, \quad m \rightarrow \infty, \quad \forall p \in P_{n}^{2}
$$

and hence

$$
\|p\|_{K} \leq(1+\epsilon)\|p\|_{Y}, \quad \forall p \in P_{n}^{2}
$$

\section{References}

[1] T. Bloom, L.P. Bos, J.-P. Calvi and N. Levenberg, Polynomial interpolation and approximation in $\mathbb{C}^{d}$, Annal. Polon. Math., 106(2012), 53-81.

[2] L. Bos, S. De Marchi, A. Sommariva and M. Vianello Weakly Admissible Meshes and Discrete Extremal Sets, Numer. Math. Theory Methods Appl., 4 (2011), 1-12. 
[3] J.P. Calvi and N. Levenberg, Uniform approximation by discrete least squares polynomials, J. Approx. Theory, 152 (2008), 82-100.

[4] S. De Marchi, M. Marchioro and A. Sommariva Polynomial approximation and cubature at approximate Fekete and Leja points of the cylinder, Appl. Math. Comput., 218 (2012), 1061710629.

[5] K. Jetter, J. Stöckler and J. D. Ward, Error Estimates for Scattered Data Interpolation, Math. Comp., 68 (1999), 733-747.

[6] F. John, Extremum problems with inequalities as subsidiary conditions, Courant Anniversary Volume (1948), Interscience Publishers, New York, 187-204.

[7] A. Kroó, Markov type inequalities for surface gradients of multivariate polynomials, J. Approx. Theory, 118 (2002), 235-245.

[8] A. Kroó, On optimal polynomial meshes, J. Approx. Theory, 163 (2011), 1107-1124.

[9] A. Kroó, Bernstein type inequalities on star-like domains in $\mathbb{R}^{d}$ with application to norming sets, Bull. Math. Sci., 3 (2013), 349361.

[10] F. Piazzon, Optimal Polynomial Admissible Meshes on Some Classes of Compact Subsets of $\mathbb{R}^{d}$, J. Approx. Theory, 207 (2016), 241-264. 